\title{
Work Plan for Determining the Occurrence of Glyphosate, Its Transformation Product AMPA, Other Herbicide Compounds, and Antibiotics in Midwestern United States Streams, 2002
}

By W.A. Battaglin, E.M. Thurman, D.W. Kolpin, E.A. Scribner, M.W. Sandstrom, and K.M. Kuivila

U.S. GEOLOGICAL SURVEY

Open-File Report 03-69

Prepared in cooperation with the U.S. ENVIRONMENTAL PROTECTION AGENCY OFFICE OF PESTICIDE PROGRAMS 


\title{
U.S. DEPARTMENT OF THE INTERIOR GALE A. NORTON, Secretary
}

\author{
U.S. GEOLOGICAL SURVEY \\ Charles G. Groat, Director
}

The use of firm, trade, and brand names in this report is for identification purposes only and does not constitute endorsement by the U.S. Geological Survey.

For additional information write to:

District Chief

U.S. Geological Survey

Box 25046, Mail Stop 415

Denver Federal Center

Denver, CO 80225-0046
Copies of this report can be purchased from:
U.S. Geological Survey
Information Services
Box 25286
Denver Federal Center
Denver, CO 80225 


\section{CONTENTS}

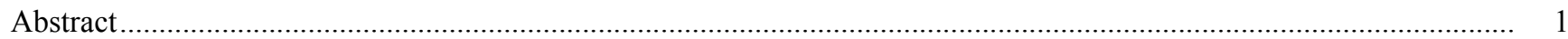

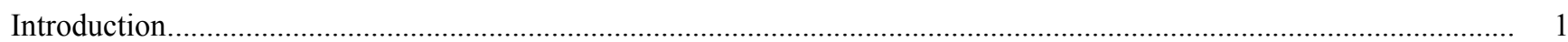

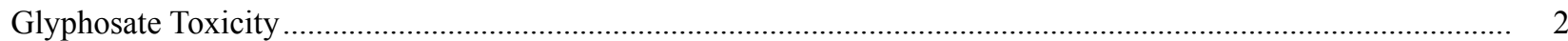

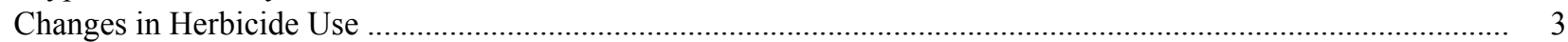

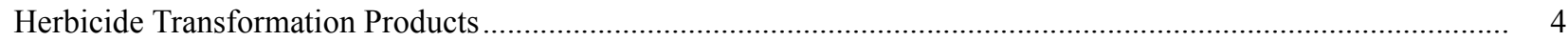



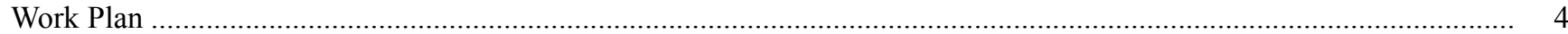

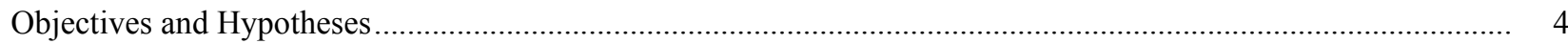

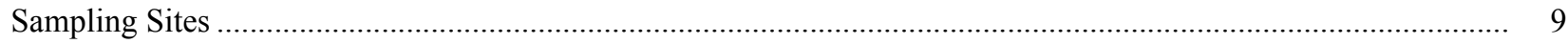

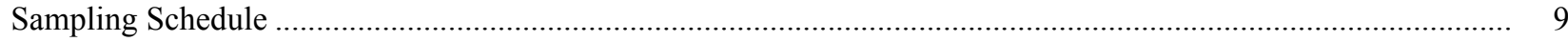

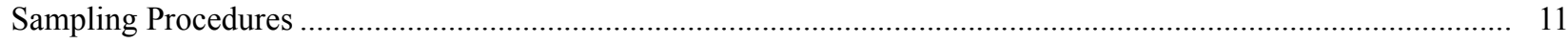

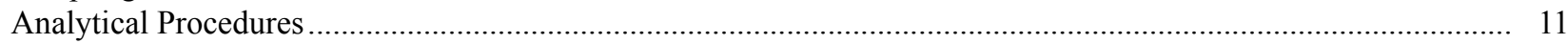

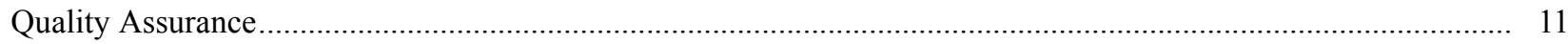

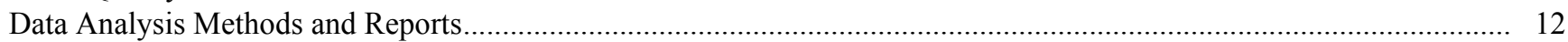

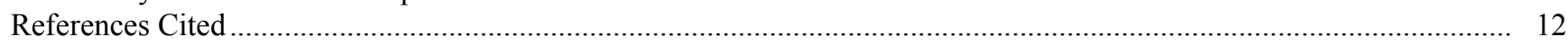

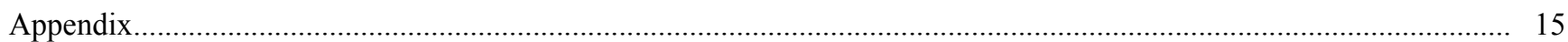

\section{FIGURES}

1. Graph showing herbicide use estimates in millions of pounds of active ingredient applied ............................... 3

2. Map showing locations of sampling sites and associated contributing drainage areas......................................... 10

\section{TABLES}

1. Toxicity of technical-grade glyphosate to aquatic organisms ................................................................... 2

2. Midwestern streams to be sampled in 2002, and streamflow conditions for samples collected in $1998 \ldots \ldots \ldots \ldots \ldots . . . . . .5$



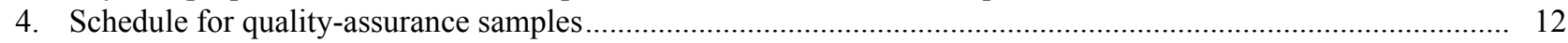

CONVERSION FACTORS AND ABBREVIATIONS

\begin{tabular}{rll}
\hline Multiply & By & To obtain \\
\hline cubic foot per second $\left(\mathrm{ft}^{3} / \mathrm{s}\right)$ & 0.02832 & cubic meter per second \\
mile $(\mathrm{mi})$ & 1.609 & kilometer \\
pound $(\mathrm{lb})$ & 453.6 & grams \\
pound per acre $(\mathrm{lb} / \mathrm{acre})$ & 1.121 & kilogram per hectare \\
square mile $\left(\mathrm{mi}^{2}\right)$ & 2.590 & square kilometer \\
gallon $(\mathrm{G})$ & 3.785 & liter $(\mathrm{L})$ \\
milligrams per liter $(\mathrm{mg} / \mathrm{L})$ & 1,000 & micrograms per liter $(\mu g / \mathrm{L})$ \\
\hline
\end{tabular}


Abbreviated Water-Quality Units

cubic meters per second $\left(\mathrm{m}^{3} / \mathrm{s}\right)$

micrograms per liter $(\mu \mathrm{g} / \mathrm{L})$

milligrams per liter $(\mathrm{mg} / \mathrm{L})$

milliliter $(\mathrm{mL})$

millimeter $(\mathrm{mm})$

microsiemens per centimeter at 25 degrees Celsius $(\mu \mathrm{S} / \mathrm{cm})$

\section{Miscellaneous Abbreviations and Acronyms}

AMPA - amino methyl phosphonic acid

DEA - deethylatrazine

DDA - didealkylatrazine

DIA - deisopropylatrazine

GCS - GCMS analysis method for herbicides and herbicide transformation products

LCGY - LCMS analysis method for glyphosate, glufosinate, and AMPA

$\mathrm{LC}_{50}$ - contaminant concentration at which 50 percent of test organisms die

LCAA - LCMS analysis method for herbicide transformation products

LCAN - LCMS analysis method for antibiotics

LCEA - LCMS analysis method for herbicides and herbicide transformation products

LCMS - Liquid chromatography mass spectrometry

LOEC - lowest observed effect concentration

NASQAN - National Stream Quality Accounting Network

NAWQA - National Water-Quality Assessment

NOEC - no observed effect concentration 


\title{
Work Plan for Determining the Occurrence of Glyphosate, Its Transformation Product AMPA, Other Herbicide Compounds, and Antibiotics in Midwestern United States Streams, 2002
}

\author{
By W.A. Battaglin, E.M. Thurman, D.W. Kolpin, E.A. Scribner, M.W. Sandstrom, and \\ K.M. Kuivila
}

\begin{abstract}
Changes in herbicide use in the Midwestern United States have been substantial over the last 5 years. Most significant is a tripling in the use of glyphosate (N-[phosphonomethyl]glycin). Over this same time period (1997-2001), atrazine use increased by 20 percent and acetochlor use increased by 10 percent, while cyanazine use decreased by 99 percent, alachlor use decreased by 70 percent, and metolachlor use decreased by 55 percent. Previous studies have documented that herbicide flushes occur in midwestern streams during runoff events for several weeks to several months following application, and that herbicide concentrations in midwestern streams during flushing events are related to rates of herbicide use.

The objective of this study is to determine the distribution of glyphosate and its primary transformation product aminomethylphosphonic acid (AMPA) in midwestern streams during postapplication and harvest-season runoff events. Water samples will be collected in 2002 during two post-herbicide-application runoff events and one harvest-season runoff event from 53 sites on streams in the Midwestern United States. All samples will be analyzed at the U.S. Geological Survey Organic Geochemistry Research Laboratory in Lawrence, Kansas, for glyphosate and 20 other herbicides. Samples will also be analyzed for a glyphosate transformation product (AMPA) and 26 other herbicide transformation
\end{abstract}

products, using GC/MS or HPLC/MS. Selected samples will be analyzed for 36 antibiotics or antibiotic transformational products. Results from this study will represent the first broad-scale investigation of glyphosate and AMPA in U.S. water resources.

\section{INTRODUCTION}

This report describes a plan of study to determine the distribution of glyphosate and its primary transformation product aminomethylphosphonic acid (AMPA) during runoff events in midwestern streams. The study will also determine if recent changes in herbicide use are affecting herbicide concentrations, and the distribution of didealkylatrazine (DDA), other herbicide transformation products, and selected antibiotics in midwestern streams.

Since 1989, the U.S. Geological Survey (USGS) Toxic Substances Hydrology Program has conducted reconnaissance studies of streams in the Midwestern United States (U.S.) to determine the geographic and seasonal distribution of herbicide compounds. Studies in 1989 and 1990 documented that large amounts of atrazine, cyanazine, alachlor, and metolachlor were flushed into streams during post-herbicide-application runoff events (Thurman and others, 1991, 1992; Goolsby and Battaglin, 1993). Additional studies in 1990-1992 using automatic samplers (Scribner and others, 1994) showed that the peak herbicide concentrations tend to occur during the first runoff event after herbicide application. Smaller herbicide concentration peaks continue to occur during runoff events for 
several weeks to several months following application. By late summer, herbicide concentrations are generally low (less than $0.05 \mu \mathrm{g} / \mathrm{L}$ ) and tend to remain low until the process repeats during the following planting season.

Recent studies have focused on collecting water samples during these post-application runoff events. The same 53 stream sites have been sampled in 1989, 1990, 1994, 1995, and 1998. Results from these studies demonstrate that the concentrations of several herbicides in midwestern streams have decreased since 1989 (Battaglin and Goolsby, 1999; Scribner and others, 2000). In some cases, these decreases correspond to declines in herbicide application amounts, whereas in other cases, the reason for the decrease more likely is changes in application practices. These studies also found a widespread occurrence of herbicide transformation products in midwestern streams. In the 1998 stream reconnaissance, the median concentrations of acetochlor, alachlor, and metolachlor were all less than the median concentration of one or more of their transformation products. This concentration pattern would be expected later in the summer during low-flow conditions but was not expected during higher flows in spring and early summer (Battaglin and others, 2001).

Glyphosate (N-[phosphonomethyl]glycin) is a broad-spectrum nonselective systemic herbicide that was first used in the early 1970's. Glyphosate is among the most widely used herbicides in the world. Glyphosate has not been extensively monitored in the
United States even though it is commonly used for agricultural, silvicultural, and nonagricultural weed control. In Germany, both glyphosate and its primary transformation product (AMPA) have been detected at low concentrations in rivers. Glyphosate, however, is both highly polar and water-soluble, making analysis for it in water samples difficult (Skark and others, 1998). In 1997 (the last year with available estimates), more than 50 million pounds of glyphosate were sold for agricultural and nonagricultural weed control (Aspelin and Grube, 1999).

\section{Glyphosate Toxicity}

Glyphosate is a nonselective herbicide that inhibits aromatic amino acid biosynthesis. It is considered to be only slightly toxic to birds, fish, and aquatic invertebrates and does not bioaccumulate. The toxicity of chemicals to nontarget organisms can be compared using various endpoints including $\mathrm{LC}_{50}$ values (concentration that kills 50 percent of test organisms), NOEC (no observed effect concentration), and LOEC (lowest observed effect concentration). The 48 -hour $\mathrm{LC}_{50}$ values for freshwater fish and aquatic invertebrates range from 55 to $780 \mathrm{mg} / \mathrm{L}$ (table 1). The LOEC's for freshwater green algal growth were less at 4.00 and $1.55 \mathrm{mg} / \mathrm{L}$, respectively. Several studies examined the effect of glyphosate on soil bacteria and found no adverse effects on soil microbial biomass or on nitrification; but in fact, microbial activity

Table 1. Toxicity of technical-grade glyphosate to aquatic organisms

$\left[\mathrm{LC}_{50}, 50\right.$ percent lethal concentration; LOEC, lowest observed effect concentration; FETAX, Frog Embryo Teratogenesis Assay; NOEC, no observed effect concentration]

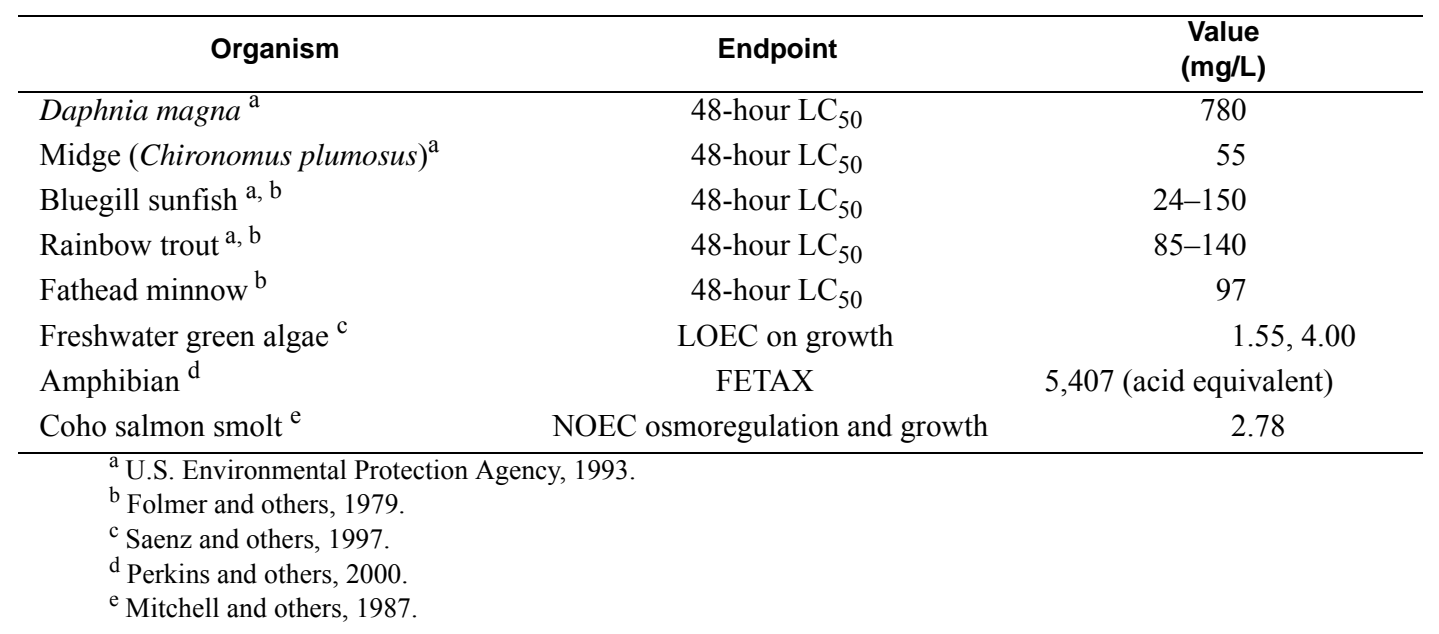


increased with glyphosate as a carbon source (Stratton, 1990; Stratton and Stewart, 1991; Busse and others, 2001; Haney and others, 2002). The effect of glyphosate on amphibians was evaluated using the Frog Embryo Teratogenesis Assay (FETAX), and the resulting $\mathrm{LC}_{50}$ was $5,407 \mathrm{mg}$ acid equivalent/L (Perkins and others, 2000). Mitchell and others (1987) found the NOEC for sublethal effects of glyphosate on coho salmon smolt osmoregulation and growth was $2.78 \mathrm{mg} / \mathrm{L}$. Some of the surfactants in glyphosate formulations, particularly the ethylated amines and contaminants associated with them, are thought to be more toxic than glyphosate.

\section{Changes in Herbicide Use}

Several substantial changes in herbicide use have occurred since the last stream reconnaissance study in 1998. The most significant is the rapid increase in the use of glyphosate that corresponded with the introduction of "Roundup Ready" crops. Between 1997 and 2001 (the newest data available) agricultural glyphosate use in nine Midwestern States (Illinois, Indiana, Iowa, Kansas, Minnesota, Missouri, Nebraska, Ohio, and Wisconsin) more than tripled from 10.3 to 36.1 million pounds applied (fig. 1). Over this same time period, atrazine use increased from 43.7 to 52.2 million pounds, acetochlor use increased from 26.1 to 28.7 million pounds, and simazine use increased from 0.8 to 1.3 million pounds; while cyanazine use decreased from 15.7 to 0.05 million pounds, alachlor use decreased from 7.8 to 2.3 million pounds, metolachlor use decreased from 46.1 to 20.5 million pounds, and metribuzin use decreased from 1.2 to 0.2 million pounds (U.S. Department of Agriculture, 2002).

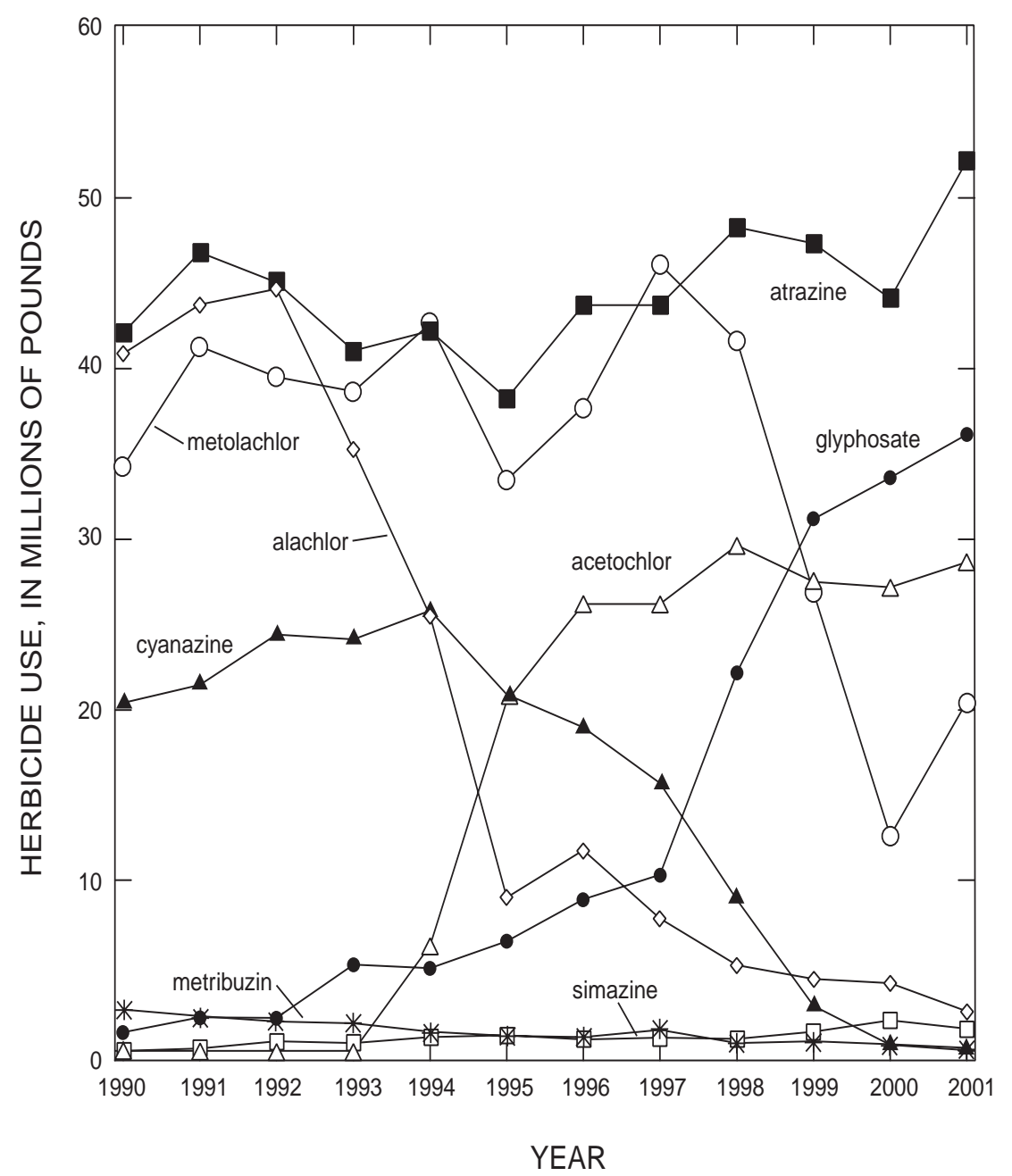

Figure 1. Herbicide use estimates in millions of pounds of active ingredient applied. 
Assuming that these trends in herbicide use continued through 2002, we suspect glyphosate and its primary transformation product AMPA will be detected in a majority of midwestern streams during 2002 post-application runoff events. We also suspect that when compared with the distribution of concentrations measured in the 53 midwestern streams in 1998, those measured in 2002 will be about the same for atrazine and acetochlor and significantly lower for alachlor, cyanazine, and metolachlor.

\section{Herbicide Transformation Products}

The half-life of glyphosate in soils typically found in the Midwestern United States is approximately 25-40 days. Glyphosate can be transformed (degraded) in soil, water, and plants to AMPA. Glyphosate loses an acetic acid moiety to form the AMPA transformation product. AMPA retains the phosphate group and many of the physical properties of glyphosate. AMPA is soluble and easily dissolved but will precipitate in the presence of iron and calcium after formation of insoluble phosphate salts (Royal Society of Chemistry, 1998). AMPA is most likely more persistent than glyphosate and also should be monitored as part of any study of glyphosate.

The 1998 reconnaissance (Battaglin and others, 2001) and other recent investigations (Thurman and others, 1994; Boyd, 2000; Kalkhoff and others, 1998; Phillips and others, 1999; Porter and others, 2001) have documented the widespread occurrence of herbicide transformation products in water. Still, scientists have not analyzed natural water samples for many of the herbicide transformation products that are likely to occur in the environment. Thurman and others (1994) showed that both the deethylatrazine (DEA) and deisopropylatrazine (DIA) transformation products of atrazine are commonly found in soils, ground water, and surface water of the Midwestern United States. Both DEA and DIA form by losing either an ethyl or isopropyl group - which is a common degradation pathway in soil. It is hypothesized that the continued transformation of DEA and DIA with the loss of either the remaining ethyl or isopropyl group will result in didealkylatrazine (DDA). Its occurrence was suspected in the past, but methods were not available until now to isolate and identify DDA. DDA retains the chlorine atom and probably retains some of the herbicidal activity and toxicity of the parent atrazine.
No published data exist for the occurrence of DDA in surface or ground water. The authors hypothesize that both AMPA and DDA will be detected in most midwestern streams.

\section{Antibiotics in Streams}

Recent studies have shown antibiotics to be present in U.S. streams (Kolpin and others, 2002). A number of the antibiotics found are those used in livestock production, but the transport mechanisms of antibiotics to streams are not yet understood. Samples collected during runoff events in midwestern streams will help to identify the dominant source of antibiotics in streams. If point sources such as sewage-treatment discharges or confined animal feeding operation (CAFO) discharges are the dominant source, then concentrations should be much lower during runoff events than during lower flow conditions. If nonpoint sources (such as manure applications to fields) are dominant, then concentrations should be about the same or higher during runoff events. Because manure applications also take place during the post-harvest period, fall and early winter storms may be "flushing" events for antibiotics.

\section{WORK PLAN}

Approximately 180 samples will be collected during the 2002 reconnaissance study. Samples will be collected from 53 sites (table 2) on small to mediumsized midwestern streams. All 2002 reconnaissance samples will be sent to the USGS Organic Geochemistry Research Laboratory (OGRL) in Lawrence, Kansas, and analyzed for 21 herbicides, 27 herbicide transformation products, and 36 antibiotics (table 3 ). The analytical reporting limits for all compounds are at or below known biologically significant levels (Battaglin and Fairchild, 2002).

\section{Objectives and Hypotheses}

The principal objective of this study is to determine the distribution of glyphosate and its primary transformation product AMPA in midwestern streams during runoff events. The proposed study also provides a plan to determine (1) if recent changes in 
Table 2. Midwestern streams to be sampled in 2002, and streamflow conditions for samples collected in 1998

$\left[\mathrm{mi}^{2}\right.$, square miles; $\mathrm{ft}^{3} / \mathrm{s}$, cubic feet per second $]$

\begin{tabular}{|c|c|c|c|c|c|c|c|c|}
\hline \multirow{3}{*}{$\begin{array}{c}\text { Map } \\
\text { no. } \\
1\end{array}$} & \multirow{3}{*}{$\begin{array}{c}\text { State } \\
\text { IA }\end{array}$} & \multirow{2}{*}{ ID } & \multirow{2}{*}{ Station name } & \multirow{2}{*}{$\begin{array}{c}\text { Drainage } \\
\text { area } \\
\left(\mathrm{mi}^{2}\right)\end{array}$} & \multicolumn{4}{|c|}{ Sampling dates and flow, in $\mathrm{ft}^{3} / \mathrm{s}$} \\
\hline & & & & & \multicolumn{2}{|c|}{ Pre-emergence } & \multicolumn{2}{|c|}{ Post-emergence } \\
\hline & & 05411850 & Turkey River near Eldorado & 640 & \multicolumn{2}{|c|}{ not sampled in 1998} & & \\
\hline 2 & IA & 05421000 & Wapsipinicon River at Independence & 1,048 & $5 / 27$ & 595 & $6 / 20$ & 1,300 \\
\hline 3 & IA & 05455100 & Old Mans Creek near Iowa City & 201 & $6 / 10$ & 209 & $6 / 30$ & 840 \\
\hline 4 & IA & 05472500 & N. Skunk River near Sigourney & 730 & $5 / 21$ & 1,140 & $6 / 10$ & 1,810 \\
\hline 5 & IA & 05474000 & Skunk River at Augusta & 4,303 & $5 / 26$ & 15,600 & $6 / 18$ & 14,400 \\
\hline 6 & IA & 05480500 & Des Moines River at Fort Dodge & 4,190 & $5 / 16$ & 7,880 & $6 / 12$ & 5,190 \\
\hline 7 & IA & 05484500 & Raccoon River at Van Meter & 3,441 & $5 / 17$ & 6,330 & $6 / 10$ & 9,160 \\
\hline 8 & IA & 06606600 & Little Sioux River at Correctionville & 2,500 & $5 / 27$ & 1,400 & $6 / 18$ & 2,760 \\
\hline 9 & IA & 06607200 & Maple River at Mapleton & 669 & $5 / 29$ & 891 & $6 / 9$ & 889 \\
\hline 10 & IA & 06609500 & Boyer River at Logan & 871 & $5 / 22$ & 1,070 & $6 / 9$ & 2,890 \\
\hline 11 & IL & 03378000 & Bonpas Creek at Browns & 228 & $5 / 23$ & 834 & $7 / 7$ & 499 \\
\hline 12 & IL & 03381495 & Little Wabash River at Carmi & 3,088 & $5 / 23$ & 9,340 & $7 / 9$ & 4,020 \\
\hline 13 & IL & 05439500 & S. Branch Kishwaukee R. at Fairdale & 387 & $5 / 20$ & 1,620 & $7 / 29$ & 61 \\
\hline 14 & IL & 05526000 & Iroquois River near Chebanse & 2,091 & $6 / 10$ & 1,340 & $7 / 8$ & 8,490 \\
\hline 15 & IL & 05540500 & Dupage River near Shorewood & 324 & $6 / 9$ & 284 & $7 / 29$ & 166 \\
\hline 16 & IL & 05569500 & Spoon River at London Mills & 1,072 & $5 / 20$ & 1,270 & $7 / 1$ & 1,780 \\
\hline 17 & IL & 05576500 & Sangamon River at Riverton & 2,618 & $5 / 26$ & 6,800 & $7 / 9$ & 4,070 \\
\hline 18 & IL & 05587000 & Macoupin Creek near Kane & 868 & \multicolumn{2}{|c|}{ not sampled in 1998} & & \\
\hline 19 & IL & 05592100 & Kaskaskia River near Cowden & 1,330 & $5 / 20$ & 1,400 & $7 / 8$ & 2,700 \\
\hline 20 & IL & 05594000 & Shoal Creek near Breese & 735 & $5 / 22$ & 768 & $7 / 8$ & 761 \\
\hline 21 & IN & 03275000 & Whitewater River near Alpine & 522 & $5 / 26$ & 727 & $7 / 8$ & 652 \\
\hline 22 & IN & 03302800 & Blue River at Fredericksburg & 283 & $5 / 27$ & 447 & $7 / 8$ & 490 \\
\hline 23 & IN & 03328500 & Eel River near Logansport & 789 & $5 / 21$ & 701 & $6 / 30$ & 688 \\
\hline 24 & IN & 03333450 & Wildcat Creek near Jerome & 146 & $5 / 21$ & 126 & $6 / 30$ & 563 \\
\hline 25 & IN & 03335000 & Wildcat Creek near Lafayette & 794 & $5 / 20$ & 883 & $7 / 1$ & 1,130 \\
\hline 26 & IN & 03351000 & White River near Nora & 1,219 & $5 / 28$ & 1,260 & $7 / 1$ & 1,780 \\
\hline 27 & $\mathrm{IN}$ & 03362500 & Sugar Creek near Edinburgh & 474 & $5 / 26$ & 1,610 & $7 / 20$ & 1,490 \\
\hline 28 & IN & 03371500 & E. Fork White River near Bedford & 3,861 & $5 / 28$ & 14,300 & $7 / 6$ & 6,050 \\
\hline 29 & $\mathrm{KS}$ & 06885500 & Black Vermillion River near Frankfort & 410 & $5 / 5$ & 80 & $6 / 9$ & 162 \\
\hline 30 & KS & 06890100 & Delaware River near Muscotah & 431 & $5 / 5$ & 111 & $6 / 10$ & 100 \\
\hline 31 & $\mathrm{MN}$ & 05317000 & Cottonwood River near New Ulm & 1,280 & $5 / 26$ & 525 & $7 / 21$ & 673 \\
\hline 32 & MN & 05476000 & Des Moines River at Jackson & 1,220 & $5 / 26$ & 339 & $8 / 24$ & 267 \\
\hline 33 & $\mathrm{MN}$ & 05320270 & Little Cobb River near Beauford & 130 & $5 / 18$ & 166 & $8 / 17$ & 28 \\
\hline 34 & MO & 06817700 & Nodaway River near Graham & 1,320 & $6 / 18$ & 5,030 & $8 / 19$ & 282 \\
\hline 35 & $\mathrm{NE}$ & 06803000 & Salt Creek at Roca & 167 & $5 / 15$ & 470 & $6 / 10$ & 148 \\
\hline 36 & $\mathrm{NE}$ & 06804000 & Wahoo Creek at Ithaca & 271 & $5 / 15$ & 118 & $6 / 8$ & 495 \\
\hline 37 & $\mathrm{NE}$ & 06880800 & W. Fork Big Blue River near Dorchester & 1,206 & $5 / 23$ & 247 & $6 / 10$ & 231 \\
\hline 38 & $\mathrm{NE}$ & 06815000 & Big Nemaha River at Falls City & 1,340 & $5 / 26$ & 333 & $6 / 8$ & 438 \\
\hline
\end{tabular}


Table 2. Midwestern streams to be sampled in 2002, and streamflow conditions for samples collected in 1998-Continued $\left[\mathrm{mi}^{2}\right.$, square miles; $\mathrm{ft}^{3} / \mathrm{s}$, cubic feet per second]

\begin{tabular}{|c|c|c|c|c|c|c|c|c|}
\hline \multirow{3}{*}{$\begin{array}{l}\text { Map } \\
\text { no. } \\
39\end{array}$} & \multirow{3}{*}{$\begin{array}{l}\text { State } \\
\mathrm{NE}\end{array}$} & \multirow{3}{*}{$\begin{array}{c}\text { ID } \\
06882000\end{array}$} & \multirow{3}{*}{$\begin{array}{c}\text { Station name } \\
\text { Big Blue River at Barneston }\end{array}$} & \multirow{3}{*}{$\begin{array}{c}\begin{array}{c}\text { Drainage } \\
\text { area } \\
\left(\mathbf{m i}^{\mathbf{2}}\right)\end{array} \\
4,447\end{array}$} & \multicolumn{4}{|c|}{ Sampling dates and flow, in $\mathrm{ft}^{3} / \mathrm{s}$} \\
\hline & & & & & \multicolumn{2}{|c|}{ Pre-emergence } & \multicolumn{2}{|c|}{ Post-emergence } \\
\hline & & & & & $5 / 15$ & 1,110 & $6 / 9$ & 1,170 \\
\hline 40 & $\mathrm{NE}$ & 06884000 & Little Blue River near Fairbury & 2,350 & $5 / 12$ & 261 & $6 / 8$ & 239 \\
\hline 41 & $\mathrm{OH}$ & 03157000 & Clear Creek near Rockbridge & 89 & $6 / 10$ & 36 & $6 / 30$ & 84 \\
\hline 42 & $\mathrm{OH}$ & 03219500 & Scioto River near Prospect & 567 & $6 / 2$ & 128 & $6 / 29$ & 1,040 \\
\hline 43 & $\mathrm{OH}$ & 03223000 & Olentangy River at Claridon & 157 & $6 / 9$ & 13 & $6 / 29$ & 3,770 \\
\hline 44 & $\mathrm{OH}$ & 03230500 & Big Darby Creek at Darbyville & 534 & $6 / 3$ & 208 & $6 / 29$ & 11,400 \\
\hline 45 & $\mathrm{OH}$ & 03234500 & Scioto River at Higby & 5,131 & $6 / 4$ & 2,620 & $7 / 8$ & 5,030 \\
\hline 46 & $\mathrm{OH}$ & 03240000 & Little Miami River near Oldtown & 129 & $6 / 10$ & 97 & $6 / 30$ & 191 \\
\hline 47 & $\mathrm{OH}$ & 03267900 & Mad River at Eagle City & 310 & $6 / 10$ & 228 & $6 / 30$ & 454 \\
\hline 48 & $\mathrm{OH}$ & 04185000 & Tiffin River at Stryker & 410 & $6 / 1$ & 98 & $7 / 7$ & 40 \\
\hline 49 & $\mathrm{OH}$ & 04186500 & Auglaize River near Fort Jennings & 332 & $6 / 17$ & 670 & $7 / 8$ & 751 \\
\hline 50 & WI & 04087240 & Root River at Racine & 190 & $6 / 1$ & 98 & $8 / 3$ & 5 \\
\hline 51 & WI & 05340500 & St. Croix River at St. Croix Falls & 6,240 & $6 / 3$ & 4,220 & $8 / 5$ & 1,690 \\
\hline 52 & WI & 05407000 & Wisconsin River at Muscoda & 10,400 & $6 / 16$ & 12,900 & $8 / 7$ & 8,890 \\
\hline 53 & WI & 05430500 & Rock River at Afton & 3,340 & $6 / 17$ & 2,140 & $7 / 21$ & 1,830 \\
\hline
\end{tabular}

Table 3. Physical properties and chemical compounds to be determined on samples collected in 2002

$\left[\mathrm{ft}^{3} / \mathrm{s}\right.$, cubic feet per second; $\mu \mathrm{S} / \mathrm{cm}$, microsiemens per centimeter at 25 degrees Celsius;

${ }^{\circ} \mathrm{C}$, degrees Celsius; mg/L, milligrams per liter; $\mu \mathrm{g} / \mathrm{L}$, micrograms per liter]

\begin{tabular}{|c|c|c|}
\hline Property or compound & Method & $\begin{array}{l}\text { Reporting } \\
\text { limit }\end{array}$ \\
\hline \multicolumn{3}{|c|}{ Physical properties } \\
\hline streamflow & meter or rating & $0.01 \mathrm{ft}^{3} / \mathrm{s}$ \\
\hline specific conductance & meter & $1.0 \mu \mathrm{S} / \mathrm{cm}$ \\
\hline $\mathrm{pH}$ & electrometric & 0.1 unit \\
\hline water temperature & thermometer or thermister & $0.1^{\circ} \mathrm{C}$ \\
\hline \multicolumn{3}{|c|}{ Herbicides } \\
\hline acetochlor & GCS & $0.05 \mu \mathrm{g} / \mathrm{L}$ \\
\hline alachlor & GCS & $0.05 \mu \mathrm{g} / \mathrm{L}$ \\
\hline ametryn & GCS & $0.05 \mu \mathrm{g} / \mathrm{L}$ \\
\hline atrazine & GCS, LCEA & $0.05 \mu \mathrm{g} / \mathrm{L}$ \\
\hline cyanazine & GCS, LCEA & $0.05 \mu \mathrm{g} / \mathrm{L}$ \\
\hline dimethenamid & GCS & $0.05 \mu \mathrm{g} / \mathrm{L}$ \\
\hline diuron & LCEA & $0.05 \mu \mathrm{g} / \mathrm{L}$ \\
\hline flufenacet & GCS & $0.05 \mu \mathrm{g} / \mathrm{L}$ \\
\hline fluometuron & LCEA & $0.05 \mu \mathrm{g} / \mathrm{L}$ \\
\hline glyphosate & LCGY & $0.10 \mu \mathrm{g} / \mathrm{L}$ \\
\hline glufosinate & LCGY & $0.10 \mu \mathrm{g} / \mathrm{L}$ \\
\hline linuron & LCEA & $0.05 \mu \mathrm{g} / \mathrm{L}$ \\
\hline metolachlor & GCS & $0.05 \mu \mathrm{g} / \mathrm{L}$ \\
\hline metribuzin & GCS & $0.05 \mu \mathrm{g} / \mathrm{L}$ \\
\hline
\end{tabular}


Table 3. Physical properties and chemical compounds to be determined on samples collected in 2002-Continued

$\left[\mathrm{ft}^{3} / \mathrm{s}\right.$, cubic feet per second; $\mu \mathrm{S} / \mathrm{cm}$, microsiemens per centimeter at 25 degrees Celsius;

${ }^{\circ} \mathrm{C}$, degrees Celsius; $\mathrm{mg} / \mathrm{L}$, milligrams per liter; $\mu \mathrm{g} / \mathrm{L}$, micrograms per liter]

\begin{tabular}{|c|c|c|}
\hline Property or compound & Method & $\begin{array}{c}\text { Reporting } \\
\text { limit }\end{array}$ \\
\hline \multicolumn{3}{|c|}{ Herbicides-Continued } \\
\hline pendimethalin & GCS & $0.05 \mu \mathrm{g} / \mathrm{L}$ \\
\hline prometon & GCS & $0.05 \mu \mathrm{g} / \mathrm{L}$ \\
\hline prometryn & GCS & $0.05 \mu \mathrm{g} / \mathrm{L}$ \\
\hline propachlor & GCS & $0.05 \mu \mathrm{g} / \mathrm{L}$ \\
\hline propazine & GCS, LCEA & $0.05 \mu \mathrm{g} / \mathrm{L}$ \\
\hline simazine & GCS, LCEA & $0.05 \mu \mathrm{g} / \mathrm{L}$ \\
\hline terbutryn & GCS & $0.05 \mu \mathrm{g} / \mathrm{L}$ \\
\hline \multicolumn{3}{|c|}{ Herbicide transformation products } \\
\hline acetochlor ESA & LCAA & $0.05 \mu \mathrm{g} / \mathrm{L}$ \\
\hline acetochlor OXA & LCAA & $0.05 \mu \mathrm{g} / \mathrm{L}$ \\
\hline acetochlor SAA & LCAA & $0.05 \mu \mathrm{g} / \mathrm{L}$ \\
\hline alachlor ESA & LCAA & $0.05 \mu \mathrm{g} / \mathrm{L}$ \\
\hline alachlor OXA & LCAA & $0.05 \mu \mathrm{g} / \mathrm{L}$ \\
\hline alachlor SAA & LCAA & $0.05 \mu \mathrm{g} / \mathrm{L}$ \\
\hline AMPA & LCGY & $0.10 \mu \mathrm{g} / \mathrm{L}$ \\
\hline cyanazine acid & LCEA & $0.05 \mu \mathrm{g} / \mathrm{L}$ \\
\hline cyanazine amide & GCS, LCEA & $0.05 \mu \mathrm{g} / \mathrm{L}$ \\
\hline deethylcyanazine & LCEA & $0.05 \mu \mathrm{g} / \mathrm{L}$ \\
\hline deethylcyanazine acid & LCEA & $0.05 \mu \mathrm{g} / \mathrm{L}$ \\
\hline deethylcyanazine amide & LCEA & $0.05 \mu \mathrm{g} / \mathrm{L}$ \\
\hline deethylatrazine & GCS, LCEA & $0.05 \mu \mathrm{g} / \mathrm{L}$ \\
\hline deethylhydroxyatrazine & LCEA & $0.05 \mu \mathrm{g} / \mathrm{L}$ \\
\hline deisopropylatrazine & GCS, LCEA & $0.05 \mu \mathrm{g} / \mathrm{L}$ \\
\hline demethylfluometuron & LCEA & $0.05 \mu \mathrm{g} / \mathrm{L}$ \\
\hline desiopropylhydroxyatrazine & LCEA & $0.05 \mu \mathrm{g} / \mathrm{L}$ \\
\hline didealkylatrazine & LCEA & $0.05 \mu \mathrm{g} / \mathrm{L}$ \\
\hline dimethenamid ESA & LCAA & $0.05 \mu \mathrm{g} / \mathrm{L}$ \\
\hline dimethenamid OXA & LCAA & $0.05 \mu \mathrm{g} / \mathrm{L}$ \\
\hline flufenacet ESA & LCAA & $0.05 \mu \mathrm{g} / \mathrm{L}$ \\
\hline flufenacet OXA & LCAA & $0.05 \mu \mathrm{g} / \mathrm{L}$ \\
\hline hydroxyatrazine & LCEA & $0.05 \mu \mathrm{g} / \mathrm{L}$ \\
\hline metolachlor ESA & LCAA & $0.05 \mu \mathrm{g} / \mathrm{L}$ \\
\hline metolachlor OXA & LCAA & $0.05 \mu \mathrm{g} / \mathrm{L}$ \\
\hline propachlor ESA & LCAA & $0.05 \mu \mathrm{g} / \mathrm{L}$ \\
\hline propachlor OXA & LCAA & $0.05 \mu \mathrm{g} / \mathrm{L}$ \\
\hline
\end{tabular}

Beta lactams

$\begin{array}{lll}\text { amoxicillin } & \text { LCAN } & 0.20 \mu \mathrm{g} / \mathrm{L} \\ \text { ampicillin } & \text { LCAN } & 0.10 \mu \mathrm{g} / \mathrm{L} \\ \text { cefotaxime } & \text { LCAN } & 0.10 \mu \mathrm{g} / \mathrm{L} \\ \text { cloxacillin } & \text { LCAN } & 0.10 \mu \mathrm{g} / \mathrm{L} \\ \text { oxacillin } & \text { LCAN } & 0.10 \mu \mathrm{g} / \mathrm{L} \\ \text { penicillin } \mathrm{G} & \text { LCAN } & 0.10 \mu \mathrm{g} / \mathrm{L} \\ \text { penicillin } \mathrm{V} & \text { LCAN } & 0.10 \mu \mathrm{g} / \mathrm{L}\end{array}$


Table 3. Physical properties and chemical compounds to be determined on samples collected in 2002-Continued

$\left[\mathrm{ft}^{3} / \mathrm{s}\right.$, cubic feet per second; $\mu \mathrm{S} / \mathrm{cm}$, microsiemens per centimeter at 25 degrees Celsius;

${ }^{\circ} \mathrm{C}$, degrees Celsius; $\mathrm{mg} / \mathrm{L}$, milligrams per liter; $\mu \mathrm{g} / \mathrm{L}$, micrograms per liter]

\begin{tabular}{|c|c|c|}
\hline Property or compound & Method & $\begin{array}{l}\text { Reporting } \\
\text { limit }\end{array}$ \\
\hline & ontinued & \\
\hline \multicolumn{3}{|l|}{ Macrolides } \\
\hline erythromycin & LCAN & $0.10 \mu \mathrm{g} / \mathrm{L}$ \\
\hline lincomycin & LCAN & $0.05 \mu \mathrm{g} / \mathrm{L}$ \\
\hline roxithromycin & LCAN & $0.10 \mu \mathrm{g} / \mathrm{L}$ \\
\hline trimethoprim & LCAN & $0.05 \mu \mathrm{g} / \mathrm{L}$ \\
\hline tylosin & LCAN & $0.10 \mu \mathrm{g} / \mathrm{L}$ \\
\hline virginiamycin & LCAN & $0.10 \mu \mathrm{g} / \mathrm{L}$ \\
\hline \multicolumn{3}{|l|}{ Quinolines } \\
\hline ciprofloxacin & LCAN & $0.05 \mu \mathrm{g} / \mathrm{L}$ \\
\hline clinafloxacin & LCAN & $0.05 \mu \mathrm{g} / \mathrm{L}$ \\
\hline flumequine & LCAN & $0.05 \mu \mathrm{g} / \mathrm{L}$ \\
\hline lomefloxacin & LCAN & $0.05 \mu \mathrm{g} / \mathrm{L}$ \\
\hline norfloxacin & LCAN & $0.05 \mu \mathrm{g} / \mathrm{L}$ \\
\hline ofloxacin & LCAN & $0.05 \mu \mathrm{g} / \mathrm{L}$ \\
\hline oxolinic acid & LCAN & $0.05 \mu \mathrm{g} / \mathrm{L}$ \\
\hline sarafloxacin & LCAN & $0.05 \mu \mathrm{g} / \mathrm{L}$ \\
\hline \multicolumn{3}{|l|}{ Sulfonamides } \\
\hline sulfachlorpyridazine & LCAN & $0.05 \mu \mathrm{g} / \mathrm{L}$ \\
\hline sulfadiazine & LCAN & $0.05 \mu \mathrm{g} / \mathrm{L}$ \\
\hline sulfadimethoxine & LCAN & $0.05 \mu \mathrm{g} / \mathrm{L}$ \\
\hline sulfamerazine & LCAN & $0.05 \mu \mathrm{g} / \mathrm{L}$ \\
\hline sulfamethazine & LCAN & $0.05 \mu \mathrm{g} / \mathrm{L}$ \\
\hline sulfamethoxazole & LCAN & $0.05 \mu \mathrm{g} / \mathrm{L}$ \\
\hline sulfathiazole & LCAN & $0.05 \mu \mathrm{g} / \mathrm{L}$ \\
\hline \multicolumn{3}{|l|}{ Tetracyclines } \\
\hline chlorotetracycline & LCAN & $0.10 \mu \mathrm{g} / \mathrm{L}$ \\
\hline doxycycline & LCAN & $0.10 \mu \mathrm{g} / \mathrm{L}$ \\
\hline minocycline & LCAN & $0.20 \mu \mathrm{g} / \mathrm{L}$ \\
\hline oxytetracycline & LCAN & $0.10 \mu \mathrm{g} / \mathrm{L}$ \\
\hline tetracycline & LCAN & $0.10 \mu \mathrm{g} / \mathrm{L}$ \\
\hline \multicolumn{3}{|l|}{$\begin{array}{l}\text { Tetracycline transformation } \\
\text { products }\end{array}$} \\
\hline anhydrochlortetracycline & LCAN & $0.10 \mu \mathrm{g} / \mathrm{L}$ \\
\hline anhydrotetracycline & LCAN & $0.20 \mu \mathrm{g} / \mathrm{L}$ \\
\hline demeclocycline & LCAN & $0.10 \mu \mathrm{g} / \mathrm{L}$ \\
\hline
\end{tabular}


herbicide use are affecting herbicide concentrations in midwestern streams, (2) the distribution of DDA and other herbicide transformation products with limited environmental data, and (3) the distribution of selected antibiotics.

Specific hypotheses:

1. Glyphosate will be detected at a majority of the stream sites in post-emergence runoff samples, but not in the majority of harvest-season runoff samples.

2. AMPA will occur with a frequency and in concentrations similar to that of acetochlor ESA. The frequency of AMPA detection will be larger in harvest-season runoff samples than in post-application runoff samples.

3. The ratio of AMPA to glyphosate will be greater than one in pre-emergence and harvest-season samples but less than one in post-emergence samples.

4. The AMPA in midwestern streams is from transformation of glyphosate and not transformation of detergents in sewage-treatment plants.

5. When compared to concentrations measured in 1998 samples, those measured in post-application runoff in 2002 will be about the same for atrazine and acetochlor and significantly lower for alachlor, cyanazine, and metolachlor.

6. Didealkylatrazine (DDA) will be detected in most midwestern streams in concentrations similar to that of de-ethylatrazine.

7. The herbicide transformation product to parent ratios for alachlor, cyanazine, and metolachlor will be larger in 2002 than in 1998 because of declining use of these products.

8. Selected antibiotics will be detected in about onehalf of the 53 midwestern streams.

9. Because of the timing of spring manure applications, antibiotic concentrations will be higher in the pre-emergence runoff samples than in the post-emergence runoff samples or harvestseason runoff samples.

\section{Sampling Sites}

The sampling sites are representative of unregulated streams that drain small, agriculturally dominated watersheds in the Midwestern United States. All but 2 of the 53 stream sites sampled in this study have been sampled in the prior reconnaissance studies (Goolsby and others, 1994; Scribner and others, 1998). The Turkey River near Eldorado site (05411850) is about 15 miles downstream from the previously sampled site at Spillville (05411600). The Little Cobb River near Beauford site (05320270) was sampled in 1998 but not in the previous reconnaissance studies, which had sampled the Rock River at Luverne (06483000). The locations of the sites to be sampled and their drainage basins are shown in figure 2. Sampling stations, drainage areas, 1998 sampling dates, and streamflow are given in table 2 .

\section{Sampling Schedule}

Three samples will be collected at each site. The first set of samples will be collected after pre-emergence herbicides have been applied (usually after planting but before crop plants emerge in May or June) and following a precipitation event that produces a significant increase in streamflow. Ideally, streamflow should represent runoff conditions with flow at or above the 50 th percentile (50 percent exceeds streamflow for the period of record, published in annual USGS Water Resources Data reports). These samples will be referred to as "pre-emergence runoff samples." The second set of samples will be collected after postemergence herbicides like glyphosate have been applied (usually to the foliage of weeds in June or July), again following a precipitation event that produces runoff conditions and streamflows at or above the 50th percentile. These samples will be referred to as "post-emergence runoff samples." The third set of samples will be collected during crop harvest (usually September to November), and following a precipitation event that produces runoff conditions and rising streamflows. These samples will be referred to as "harvest-season runoff samples." 


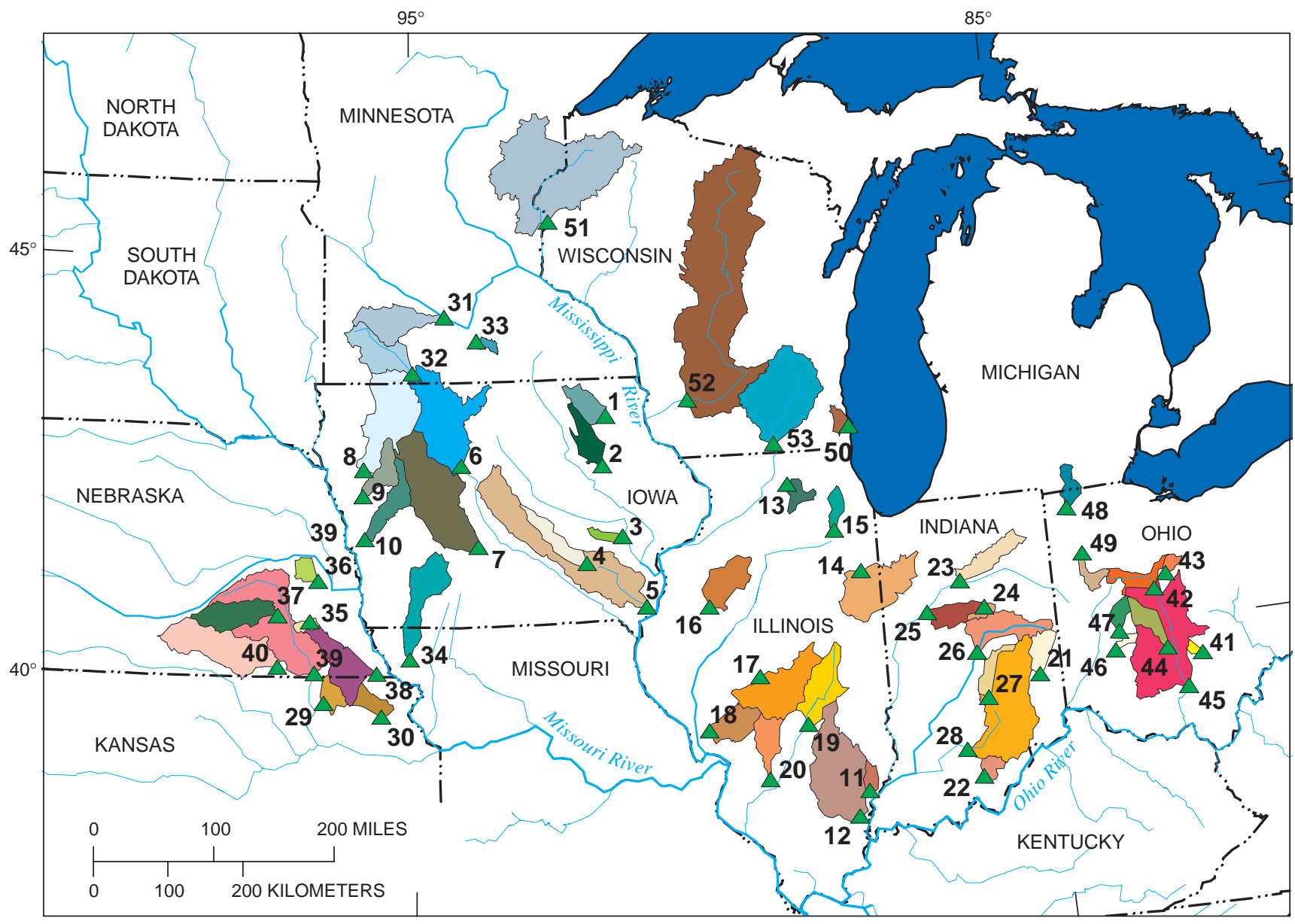

EXPLANATION
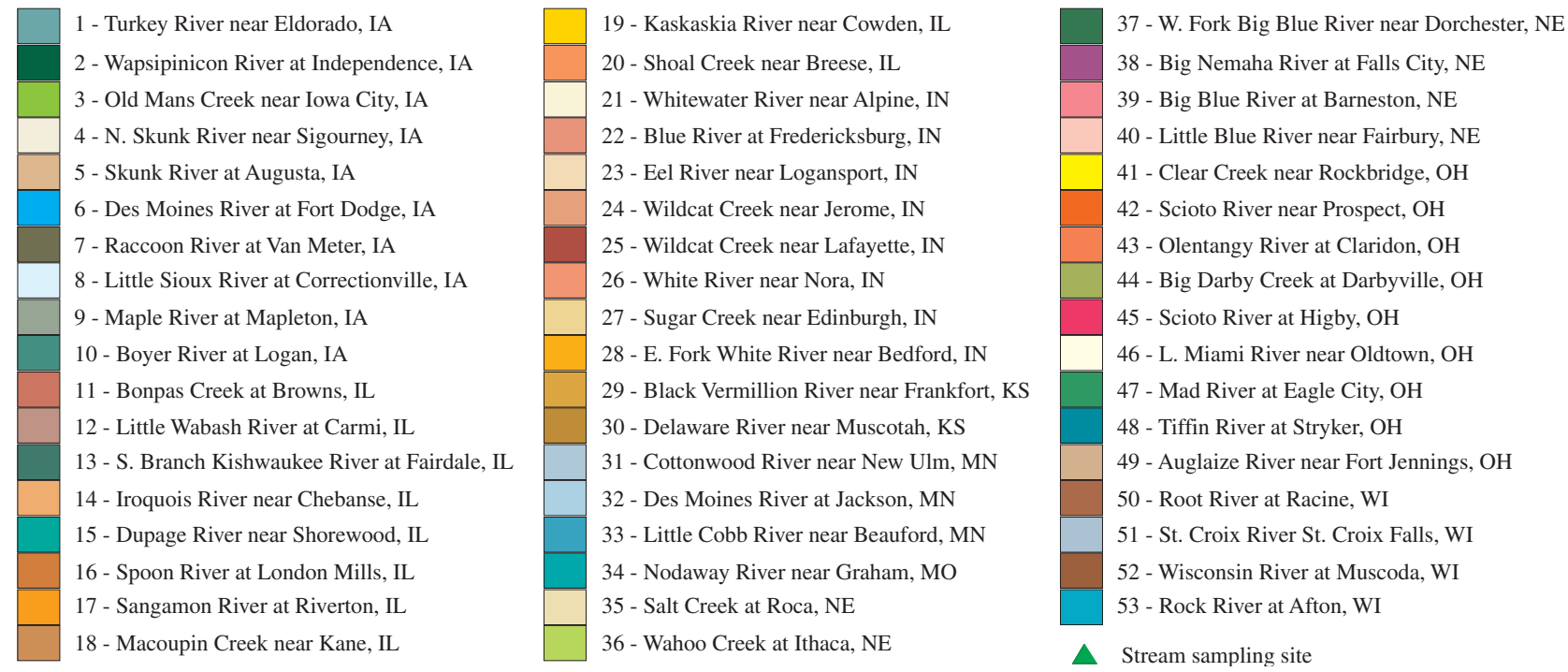

Figure 2. Locations of sampling sites and associated contributing drainage areas. 


\section{Sampling Procedures}

Samples will be collected using methods described in Shelton (1994). The equal-width-increment sampling method (Edwards and Glysson, 1988) will be used for all samples. The step-by-step sampling procedure is given in the Appendix. All pesticide samples will be filtered through $0.7-\mathrm{mm}$ pore-size baked glass-fiber filters, using an aluminum plate filter holder and a ceramic piston fluid-metering pump with all Teflon tubing, into precleaned $125-\mathrm{mL}$ amber glass bottles. Eight $125-\mathrm{mL}$ amber glass bottles from each site will be filled and sent to the ORGL. Sample bottles will be clearly labeled with waterproof marker or preprinted labels. The minimum information on the label will be the project code, site id, site name, date and time of sample collection, sampler name(s), and the following phrase "USGS 2002 RECON." Field measurements for specific conductance, $\mathrm{pH}$, and temperature will be taken for all samples and a discharge will be obtained by direct measurement, from a rating curve or estimated from a nearby USGS gaging station.

\section{Analytical Procedures}

Glyphosate and AMPA. Samples are analyzed for glyphosate, glufosinate, and AMPA by using a liquid chromatography mass spectrometry (LCMS) method as described in Lee and others (2002a). For the LCGY analysis, $10-\mathrm{mL}$ water samples are derivatized in the laboratory and extracted with solid-phase extraction (SPE). The analytes (table 3) are separated by LC and identified with an MS in electrospray negative-ion (ES-NEG) mode operating in selected-ion mode. The concentration of each sample is determined by using labeled glyphosate as an internal standard and a standard-addition replicate using the following equation:

$$
\mathrm{C}=\left[\left(\mathrm{A}_{\mathrm{c}} / \mathrm{A}_{\mathrm{i}}\right) /\left(\mathrm{A}_{\mathrm{csa}} / \mathrm{A}_{\mathrm{isa}}-\mathrm{A}_{\mathrm{c}} / \mathrm{A}_{\mathrm{i}}\right)\right] \times \mathrm{SA}_{\mathrm{c}} \times \mathrm{DF}
$$

where

$\mathrm{C}$ is concentration of the selected compound in the sample,

Ac is area of peak of the ion for the selected compound,

$A_{i}$ is area of peak for the internal standard,
$A_{c s a}$ is area of the peak for the selected compound in the standard-addition sample,

$\mathrm{A}_{\text {isa }}$ is the area of the peak for the internal standard in the standard-addition sample,

$\mathrm{SA}_{\mathrm{c}}$ is concentration of the standard addition, and $\mathrm{DF}$ is dilution factor.

Other Herbicides and Transformation Products. The other herbicide compounds and transformation products (table 3 ) are analyzed using three methods. The LCEA analysis (Lee and others, 2002b) uses SPE and atmospheric chemical ionization (APCI) positive LCMS. The LCAA analysis (Lee and others, 2001) uses SPE and ES-NEG-LCMS. The GCS analysis (Zimmerman and Thurman, 1999; Kish and others, 2000) uses SPE and gas chromatography mass spectrometry (GCMS). All three methods use 123-mL samples and include surrogate and internal standards. Mass spectrometers are operated in selected-ion mode, and concentrations of detected analytes are calculated from calibration curves.

Antibiotics. A method (LCAN) has been developed for the trace analysis (concentrations between 0.05 and $2.0 \mu \mathrm{g} / \mathrm{L}$ ) of five classes of antibiotics consisting of seven beta lactams (BLs), six macrolides (MCs), eight quinolines (QUs), seven sulfonamides (SAs), and eight tetracyclines (TCs) (table 3). These antibiotics are commonly are used by humans, livestock, and pets or are agricultural feed additives. Some of these antibiotics are suspected to leach into ground and surface water. Three slightly different sample preparation methods are used for BLs and MCs, QUs and SAs, and TCs. All samples are extracted with SPE and determined by LCMS with positive-ion electrospray using methods similar to Lindsey and others (2001). Surrogate and internal standards are used in the analysis of 10-mL samples, and concentrations of detected analytes are calculated from calibration curves acquired with the samples.

\section{Quality Assurance}

Quality assurance of sample collection and handling will consist of 15 concurrent replicate samples and 4 field blank samples (table 4). In the laboratory, 10 percent or more of the samples will be split and analyzed in replicate (including sample preparation) to yield data on the precision of the analysis. These same samples are also analyzed as spikes for 
Table 4. Schedule for quality-assurance samples

[CR, current replicates; FB, field blanks;--, none]

\begin{tabular}{crlrrr}
\hline State & ID & \multicolumn{1}{c}{ Station name } & $\begin{array}{r}\text { Pre- } \\
\text { emer- } \\
\text { gence } \\
\text { sample }\end{array}$ & $\begin{array}{r}\text { Post- } \\
\text { emer- } \\
\text { gence } \\
\text { sample }\end{array}$ & $\begin{array}{r}\text { Harvest- } \\
\text { season } \\
\text { sample }\end{array}$ \\
\hline IA & 05411600 & Turkey River near Eldorado & $\mathrm{CR}$ & -- & -- \\
IA & 05484500 & Raccoon River at Van Meter & -- & $\mathrm{CR}$ & -- \\
IA & 06607200 & Maple River at Mapleton & -- & -- & FB \\
IL & 03378000 & Bonpas Creek at Browns & -- & -- \\
IL & 03381495 & Little Wabash River at Carmi & -- & $\mathrm{CR}$ & -- \\
IL & 05592100 & Kaskaskia River near Cowden & -- & -- & $\mathrm{CR}$ \\
IN & 03275000 & Whitewater River near Alpine & FB & -- & -- \\
IN & 03362500 & Sugar Creek near Edinburgh & -- & $\mathrm{CR}$ & -- \\
IN & 03371500 & E. Fork White River near Bedford & -- & -- & $\mathrm{CR}$ \\
KS & 06885500 & Black Vermillion River near Frankfort & $\mathrm{CR}$ & -- & -- \\
MN & 05476000 & Des Moines River at Jackson & -- & $\mathrm{CR}$ & -- \\
MO & 06817700 & Nodaway River near Graham & FB & -- & -- \\
NE & 06880800 & W. Fork Big Blue River nr Dorchester & -- & $\mathrm{CR}$ & -- \\
NE & 06882000 & Big Blue River at Barneston & -- & -- & $\mathrm{CR}$ \\
OH & 03219500 & Scioto River near Prospect & $\mathrm{CR}$ & -- & -- \\
OH & 03267900 & Mad River at Eagle City & -- & $\mathrm{CR}$ & -- \\
OH & 04185000 & Tiffin River at Stryker & -- & -- & $\mathrm{CR}$ \\
WI & 04087240 & Root River at Racine & $\mathrm{CR}$ & -- & -- \\
WI & 05430500 & Rock River at Afton & -- & FB & -- \\
\hline
\end{tabular}

determining accuracy. Method blanks are prepared and analyzed with each batch of samples. Standard reference materials (SRMs), if available, are routinely used as Laboratory Control Standards (LCS).

\section{DATA ANALYSIS METHODS AND REPORTS}

Results from this study will represent the first broad-scale investigation of glyphosate and AMPA in Midwestern United States streams. Analytical results from this study will be published in a USGS open-file report. Analysis of the results of this study will be published in one or more journal articles to be prepared in 2003 and 2004. Some ancillary data in addition to streamflow will be collected in association with the water-quality data in order to better understand the information provided. The amount of ancillary data collected will be limited by resources but at a minimum will include estimates of corn and soybean planting date, monthly precipitation and temperature, crops planted (will not be available until 2002 Census of Agriculture data are available), and pesticide use (may only be State level data).

\section{REFERENCES CITED}

Aspelin A.L., and Grube, A.H., 1999, Pesticide industry sales and usage — 1996 and 1997 market estimates: U.S. Environmental Protection Agency, Office of Prevention, Pesticides, and Toxic Substances, Washington, D.C. 733-R-99-001, 39 p.

Battaglin, W.A., and Fairchild, J., 2002, Potential toxicity of pesticides measured in midwestern streams to aquatic organisms: Water Science and Technology, v. 45, no. 9, p. 95-103, available on-line at http://co.water.usgs.gov/midconherb/html/ onlinereports.html

Battaglin, W.A., Furlong, E.T., Burkhardt, M.R., and Boyer, L.L., 2001, Concentrations of selected sulfonylurea, sulfonamide, and imidazolinone herbicides, and other pesticides in storm runoff from 71 streams, outflow from 5 reservoirs, and ground water from 25 wells in 
the Midwestern United States, 1998: U.S. Geological Survey Water-Resources Investigations Report 00-4225, $123 \mathrm{p}$., available on-line at http://co.water.usgs.gov/midconherb/pdf/wrir004225.pdf

Battaglin, W.A., and Goolsby, D.A., 1999, Are shifts in herbicide use reflected in concentration changes in Midwestern Rivers? Environmental Science and Technology, v. 33, p. 2917-2925.

Boyd, R.A., 2000, Herbicides and herbicide degradates in shallow groundwater and the Cedar River near a municipal well field, Cedar Rapids, Iowa: The Science of the Total Environment, v. 248, p. 241-253.

Busse, M.D., Ratcliff, A.W., Shestak, C.J., and Powers, R.F., 2001, Glyphosate toxicity and the effects of longterm vegetation control on soil microbial communities: Soil Biology and Biochemistry, v. 33, p. 1777-1789.

Edwards, T.K., and Glysson, D.G., 1988, Field methods for measurement of fluvial sediment: U.S. Geological Survey Open-File Report 86-531, 118 p.

Ferrer, I., Thurman, E.M., and Barcelo, D., 1997, Identification of ionic chloroacetanilide-herbicide metabolites in surface water and groundwater by HPLC/MS using negative ion spray: Analytical Chemistry, v. 69, p. 4547-4553.

Folmer, L., Sanders, H., and Julin, A., 1979, Toxicity of the herbicide glyphosate and several of its formulations to fish and aquatic invertebrates: Archives of Environmental Contaminant Toxicology, v. 8, p. 269-278.

Goolsby, D.A., and Battaglin, W.A., 1993, Occurrence, distribution, and transport of agricultural chemicals in surface waters of the Midwestern United States, in Goolsby, D.A., and others, eds., Selected papers on agricultural chemicals in water resources of the Midcontinental United States: U.S. Geological Survey Open-File Report 93-418, p. 1-24.

Goolsby, D.A., Boyer, L.L., and Battaglin, W.A., 1994, Plan of study to determine the effects of changes in herbicide use on herbicide concentrations in midwestern streams, 1989-94: U.S. Geological Survey Open-File Report 94-347, 14 p.

Haney, R.L., Senseman, S.A., and Hons, F.M., 2002, Effect of Roundup Ultra on microbial activity and biomass from selected soils: Journal of Environmental Quality, v. 31, p. $730-735$.

Kalkhoff, S.J., Kolpin, D.W., Thurman, E.M., Ferrer, I., and Barcelo, D., 1998, Degradation of chloroacetanilide herbicides-The prevalence of sulfonic and oxanilic acid metabolites in Iowa groundwaters and surface waters: Environmental Science and Technology, v. 32, p. $1738-1740$.
Kish, J.L., Thurman, E.M., Scribner, E.A., and Zimmerman, L.R., 2000, Method of analysis by the U.S. Geological Survey Organic Geochemistry Research Group-Determination of triazine and chloroacetanilide herbicides in water by solid-phase extraction and capillary-column gas chromatography/mass spectrometry with selected-ion monitoring: U.S. Geological Survey Open-File Report 00-385, 13 p.

Kolpin, D.W., Furlong, E.T., Meyer, M.T., Thurman, E.M., Zaugg, S.D., Barber, L.B., and Buxton, H.T., 2002, Pharmaceuticals, hormones, and other organic wastewater contaminants in U.S. streams, 1999-2000A national reconnaissance: Environmental Science and Technology, v. 36, p. 1202-1211.

Lee, E.A., Kish, J.L., Zimmerman, L.R., and Thurman, E.M., 2001, Methods of analysis by the U.S. Geological Survey Organic Geochemistry Research GroupUpdate and additions to the determination of chloroacetanilide herbicide degradation compounds in water using high-performance liquid chromatography/mass spectrometry: U.S. Geological Survey Open-File Report 01-10, $17 \mathrm{p}$.

Lee, E.A., Strahan, A.P., and Thurman, E.M., 2002a, Methods of analysis by the U.S. Geological Survey Organic Geochemistry Research Group-Determination of glyphosate, aminomethylphosphonic acid, and glufosinate in water using online solid-phase extraction and high-performance liquid chromatography/mass spectrometry: U.S. Geological Survey Open-File Report 01-454, 13 p., available on-line at http://ks.water.usgs.gov/Kansas/pubs/abstracts/ofr.01454.abs.html

Lee, E.A., Strahan, A.P., and Thurman, E.M., 2002b, Methods of analysis by the U.S. Geological Survey Organic Geochemistry Research Group-Determination of triazine and phenylurea herbicides and their degradation products in water using solid-phase extraction and liquid chromatography/mass spectrometry: U.S. Geological Survey Open-File Report 02-436, 19 p., available on-line at http://ks.water.usgs.gov/Kansas/pubs/abstracts/of.02436.html

Lindsey, M.E., Meyer, M.T., and Thurman, E.M., 2001, Analysis of trace levels of sulfonamide and tetracycline antimicrobials in groundwater and surface water using solid-phase extraction and liquid chromatography/mass spectrometry: Analytical Chemistry, v. 74 , p. $4640-4646$.

Mitchell, D., Chapman, P., and Long, T., 1987, Seawater challenge testing of Coho salmon smelts following exposure to Roundup herbicide: Environmental Toxicology and Chemistry, v. 6, p. 875-878. 
Perkins, P.J., Boermans, H.J., and Stephenson, G.R., 2000, Toxicity of glyphosate and triclopyr using the frog embryo teratogenesis assay - Xenopus: Environmental Toxicology and Chemistry, v. 19, no. 4, p. 940-945.

Phillips, P.J., Wall, G.R., Thurman, E.M., Eckhardt, D.A., and VanHoesen, J., 1999, Metolachlor and its metabolites in tile drain and stream runoff in the Conajoharie Creek watershed: Environmental Science and Technology, v. 33, p. 3531-3537.

Porter, S.D., Harris, M.A., and Kalkhoff, S.J., 2001, Influence of national factors on the quality of Midwestern streams and rivers: U.S. Geological Survey WaterResources Investigations Report 00-4288, 13 p.

Royal Society of Chemistry, 1998, Metabolic pathways of agrochemicals - Part one, herbicides and plant growth regulators, T.R. Roberts, ed:, London, Royal Society of Chemistry Books, p. 396-400.

Saenz, M.E., DiMarzio, W.D., Alberdi, J.L., and Tortorelli, M., 1997, Effects of technical grade and a commercial formulation of glyphosate on algal population growth: Bulletin of Environmental Contaminant Toxicology, v. 59 , p. $638-644$.

Scribner, E.A., Battaglin, W.A., Goolsby, D.A., and Thurman, E.M., 2000, Changes in herbicide concentrations in midwestern streams in relation to changes in use, 1989-1998: The Science of the Total Environment, v. 248 , no. $2-3$, p. 255-263.

Scribner, E.A., Goolsby, D.A., Thurman, E.M., and Battaglin, W.A., 1998, A reconnaissance for selected herbicides, metabolites, and nutrients in streams of nine Midwestern States, 1994-95: U.S. Geological Survey Open-File Report 98-181, 44 p.

Scribner, E.A., Goolsby, D.A., Thurman, E.M., Meyer, M.T., and Pomes, M.L., 1994, Concentrations of selected herbicides, two triazine metabolites, and nutrients in storm runoff from nine stream basins in the Midwestern United States, 1990-92: U.S. Geological Survey Open-File Report 94-396, 144 p.

Shelton, L.R., 1994, Field guide for collection and processing stream-water samples for the national water-quality assessment program: U.S. Geological Survey Open-File Report 94-455, 42 p.
Skark, C., Zullei-Seibert, N., Schottler, U., and Schlett, C., 1998, The occurrence of glyphosate in surface water: International Journal of Environmental Analytical Chemistry, v. 70, no. 1-4, p. 93-104.

Stratton, G.W., 1990, Effects of the herbicide glyphosate on nitrification in four soils from Atlantic Canada: Water, Air, and Soil Pollution, v. 51, no. 373-383.

Stratton, G.W., and Stewart, K.E., 1991, Effects of the herbicide glyphosate on nitrogen cycling in an acid forest soil: Water, Air, and Soil Pollution, v. 60, p. 231-247.

Thurman, E.M., Goolsby, D.A., Meyer, M.T., and Kolpin, D.W., 1991, Herbicides in surface waters of the Midwestern United States - The effect of spring flush: Environmental Science and Technology, v. 25, p. 1794-1796.

Thurman, E.M., Goolsby, D.A., Meyer, M.T., and Kolpin, D.W., 1992, A reconnaissance study of herbicides and their metabolites in surface water of the Midwestern United States using immunoassay and gas chromatography/mass spectrometry: Environmental Science and Technology, v. 26, p. 2440-2447.

Thurman, E.M., Meyer, M.T., Mills, M.S., Zimmerman, L.R., Perry, C.A., and Goolsby, D.A., 1994, Formation and transport of deethylatrazine and deisopropylatrazine in surface water: Environmental Science and Technology, v. 28, p. 2267-2277.

U.S. Department of Agriculture, 2002, National Agricultural Statistics Service (NASS), Agricultural Chemical Use Database: Accessed 10/16/2002 at http://www.pestmanagement.info/nass/

U.S. Environmental Protection Agency, 1993, Reregistration Eligibility Decision (RED) Glyphosate: Accessed $10 / 16 / 2002$ at http://www.epa.gov/oppsrrd1/REDs/old_reds/ glyphosate.pdf

Zimmerman, L.R., and Thurman, E.M., 1999, Method of analysis by the U.S. Geological Survey Organic Geochemistry Research Group-Determination of triazine and chloroacetanilide herbicides in water by solid-phase extraction and capillary-column gas chromatography/mass spectrometry with selected-ion monitoring: U.S. Geological Survey Open-File Report 98-634, $21 \mathrm{p}$. 
APPENDIX 
16 Work Plan for Determining the Occurrence of Glyphosate, Its Transformation Product AMPA, Other Herbicide Compounds, and Antibiotics in Midwestern U.S. Streams, 2002 


\section{APPENDIX - Detailed sample collection and processing instructions}

\section{Determining where and when to sample}

1. Determine which sites are to be sampled in your State (table 2).

2. Determine which sites need quality-assurance samples collected (table 4).

3. Follow news on progress of planting and weather (Battaglin will provide weekly data during the growing season, or you can monitor at http://usda.mannlib.cornell.edu/reports/nassr/ field/weather/) accessed May, June, and July 2002.

4. Collect the first set of surface-water samples (preemergence runoff) after pre-emergence herbicides have been applied (usually May or June), when corn is at least 50 percent planted, and following a precipitation event that produces a significant increase in streamflow. Ideally, the sample should represent runoff conditions with streamflow at or above the 50th percentile, which is equal to the "50 percent exceeds" streamflow value for the period of record, published for most sites in annual USGS Water Resources Data reports.

5. Continue to follow news on local progress of plant development and weather.

6. Collect the second set of surface-water samples (post-emergence runoff) after post-emergence herbicides, particularly glyphosate, have been applied (usually June or July), when soybeans are nearing 100 percent emerged, and following a precipitation event that produces runoff conditions and streamflow at or above the 50th percentile.

7. Collect the third set of surface-water samples (harvest-season runoff) during or after crop harvest (usually September, October, or November) following a precipitation event that produces runoff conditions and a rise in streamflow at the site. Streamflow does not have to be at or above the 50th percentile.

\section{Sample processing}

1. Preclean all equipment with a Liquinox/tap-water solution, rinse with tap water, deionized water, then methanol, and then air dry.
2. Collect and composite a minimum of $\sim 2$ liters of water using equal-width-increment sampling into a large precleaned glass carboy.

3. Make field measurements of $\mathrm{pH}$, temperature, and specific conductance, in the stream if possible, or on the composite sample.

4. Filter $100 \mathrm{~mL}$ of sample water through a $0.7-\mathrm{mm}$ pore-size baked glass-fiber filter to condition, and then filter $1.0 \mathrm{~L}$ of sample water into eight $125-\mathrm{mL}$ baked amber glass bottles.

5. Label all sample bottles clearly with waterproof marker or preprinted labels (a paper label completely covered with clear packing tape is preferred). The minimum information on the label for bottles sent to the ORGL is the project code, site ID, site name, date and time of sample collection, sampler name(s), and the phrase "USGS 2002 RECON" as shown below:

\begin{tabular}{ll}
\hline Project code & DBD \\
station id & 05465500 \\
stream name/loca- & Iowa River at Wapello, IA \\
$\quad$ tion & \\
date and time & 6-18-02 @ 1015 \\
sampler & Joe Sample and Jane \\
$\quad$ name/initials & Stream \\
study label & USGS 2002 RECON \\
\hline
\end{tabular}

6. Glass bottles will be placed in foam packers to help prevent breakage. The eight bottles from each site will then be placed in a plastic bag, which will help keep them dry in the coolers and keep all bottles from the same site together. All samples are to be kept chilled on ice and shipped within 2 days to the ORGL in Lawrence, Kansas.

7. Notify Bill Battaglin (303-236-4882 extension 256) wbattagl@usgs.gov with the date and time of sample collection and physical properties as soon as possible after samples are shipped.

\section{Quality-assurance samples}

Concurrent replicate and field blank samples will be collected during all three sampling periods (table 4). The intent of these samples is to help determine analytical precision. The concurrent replicate 
sample will require collecting and compositing a larger volume of water from the sites than normally needed. The replicates will be processed in the same manner as the normal samples using the same filter if possible. Record the same site name and ID for both samples but a time that is 1 minute later for the replicate sample. Both samples will be sent to the ORGL in Lawrence, Kansas, for herbicide analysis. The field blank samples will consist of organic-free water that is processed through all of the sample collection and filtration equipment after it has been cleaned as described above. These samples will be labeled as a field blank and sent to the ORGL in Lawrence, Kansas, for analysis. 